\title{
Manejo de la exacerbación aguda severa de EPOC
} Management of severe acute exacerbations of COPD

\author{
Eder Cáceres ${ }^{(1)}$; Rubén Duenas ${ }^{(2)}$, Marcela Poveda ${ }^{(3)}$, Ricardo Buitrago ${ }^{(4)}$
}

\section{RESUMEN}

La EAEPOC (Exacerbación Aguda de Enfermedad pulmonar Obstructiva Crónica) es un motivo de consulta frecuente en los servicios de urgencias y cuando es severa usualmente requiere el manejo en una unidad de cuidados intermedios o intensivos donde se garantice un adecuado monitoreo del paciente y la provisión de asistencia ventilatoria. Se realizó una revisión de la literatura acerca de la evaluación, manejo y seguimiento de la EAEPOC severa haciendo énfasis en los fundamentos e indicaciones de la VMNI y en la evaluación del paciente una vez se ha instaurado esta terapia la cual es en la actualidad la primera opción en pacientes con EAEPOC en inminencia de o en falla ventilatoria hipercápnica y ha mostrado beneficios significativos en la recuperación y supervivencia. Es importante junto a la evaluación clínica la toma de gases arteriales en estos pacientes que permitirán un enfoque inicial y servirán posteriormente como guía en la toma oportuna de decisiones.

Palabras clave: enfermedad pulmonar obstructiva crónica, exacerbación aguda, ventilación mecánica no invasiva, CPAP, falla respiratoria.

\section{ABSTRACT}

AECOPD (acute exacerbation of chronic pulmonary disease) is a frequent motive of presentation to emergency services. When severe, it usually requires management at an intermediate or intensive care unit, where adequate monitoring and ventilatory assistance can be provided. A review was carried out of the literature on the evaluation, management, and follow-up of severe AECOPD, with emphasis on the rationale and indications of noninvasive mechanical ventilation (NIMV), as well as on the assessment of the patient once this therapy has been initiated. NIMV is currently the firstchoice treatment in patients with AECOPD with impending or actual hypercapnic ventilatory failure, and has shown significant benefits in terms of recovery and survival. It is important to supplement clinical evaluation with arterial blood gas analysis, which allows initial focusing and afterwards serves as a guideline for timely decisions.

Key words: chronic obstructive pulmonary disease, acute exacerbation, noninvasive mechanical ventilation, CPAP, respiratory failure.

Rev Colomb Neumol 2011; 23(2): 48-54.

Actualmente la definición más aceptada de una exacerbación aguda en un paciente con EPOC (Enfermedad pulmonar obstructiva crónica) es un empeoramiento sostenido en la condición de base del paciente, más allá de las variaciones diarias normales, de instauración aguda y que necesite de un cambio en la medicación regular del paciente (1). Dentro de los factores encontrados en los pacientes admitidos a hospitalización por EAEPOC (Exacerbación Aguda de EPOC) están ausencia de vacunación contra influenza, no asistencia a programas de rehabilitación pulmonar, no uso o uso subterapéutico de oxígeno domiciliario e inhaladores y tabaquismo al momento de la hospitalización (2) , a su vez estos pacientes hospitalizados sufren consecuencias a mediano y largo plazo como aumento en el riesgo de hospitalizaciones posteriores, empeoramiento de las pruebas de función pulmonar, menor duración entre exacerbaciones, deterioro de la función muscular y aumento de la mortalidad (3).

\footnotetext{
(1) Residente Cuidado Intensivo Universidad de La Sabana

(2) Neumologo clinica shaio

(3) Cardiologa Intensivista Clinica Shaio

(4) Cardiologo Intensivista Clinica Shaio
}

Correspondencia: Dr. Eder Cáceres. Correo electrónico: caceres.eder@hotmail.com

Recibido: 15 de Agosto de 2011 Aceptado: 23 de Septiembre de 2011. 
Existen varias clasificaciones de la severidad de la exacerbación de EPOC. La clasificación de la ATS/ERS tiene en cuenta el escenario en el cual se maneja la exacerbación:

- Leve: se presenta un aumento en el requerimiento de la medicación que el paciente puede manejar por sí mismo de manera ambulatoria.

- Moderada: el paciente aumenta el requerimiento de medicación y siente la necesidad de buscar asistencia médica adicional.

- Severa: paciente o cuidadores reconocen un deterioro claro y rápido en la condición del paciente con requerimiento de hospitalización.

La clasificación de Anthonisen está basada en características clínicas de la exacerbación (6) y ha sido usada ampliamente para establecer la severidad y definir conductas terapéuticas (tabla 1):

\section{EVALUACIÓN DEL PACIENTE CON EAEPOC SEVERA}

El paciente con EAEPOC severa presenta características en la historia clínica y examen físico que permiten establecer su compromiso sistémico y respiratorio. Usualmente son pacientes con comorbilidades asociadas e historia de exacerbaciones recurrentes, al examen físico presentan taquipnea, uso de músculos accesorios, respuesta irregular al manejo médico inicial, compromiso sistémico dado por inestabilidad hemodinámica, cianosis, fiebre, taquicardia, edema periférico, signos de falla cardíaca y alteración del estado mental.
Dentro de la valoración inicial del paciente con EAEPOC severa debe incluirse:

- $\quad$ Saturación arterial de oxígeno

- Gases arteriales

- $\quad$ Radiografía de tórax

- Pruebas sanguíneas

- $\quad$ Gram y cultivo de esputo

- Electrocardiograma

Adicionalmente dependiendo del contexto clínico se debe garantizar el estudio de posibles diagnósticos diferenciales: tromboembolismo pulmonar, falla cardíaca congestiva, arritmias cardíacas, neumonía.

Después de realizada la evaluación inicial la EAEPOC severa usualmente requiere manejo en una unidad de cuidado intensivo (UCI) o intermedio ya que es indispensable un seguimiento clínico estricto por parte del personal médico y de enfermería, especialmente aquellos pacientes que lleguen a requerir soporte ventilatorio. Algunas indicaciones generales para el requerimiento de manejo en $\mathrm{UCl}$ son $(9,29)$ :

- $\quad$ Respuesta irregular al manejo inicial

- Falla respiratoria o inminencia de la misma

- Inestabilidad hemodinámica

- Disfunción de otro órgano (renal, neurológico, hepático, etc.)

Tabla1. Clasificación de Anthonisen para la excerbación de EPOC (6)

\begin{tabular}{|cll|}
\hline Severidad de la Exacerbación & Tipo de Exacerbación & Características \\
\hline Severa & Tipo 1 & $\begin{array}{l}\text { Aumento de la disnea, volumen del esputo y } \\
\text { purulencia del esputo }\end{array}$ \\
Moderada & Tipo 2 & 2 de los 3 síntomas descritos \\
Leve & Tipo 3 & 1 de los 3 síntomas asociado a uno o más de los \\
& & siguientes: \\
& - Tos \\
& - Sibilancias \\
& - Aumento de frecuencia respiratoria \\
& - Aumento de frecuencia cardiaca \\
& - Fiebre \\
& - Infección respiratoria superior en los últimos 5 días
\end{tabular}




\section{TRATAMIENTO DE LA EAEPOC SEVERA}

En la EAEPOC existen dos pilares fundamentales de tratamiento que deben considerarse siempre, estos son la terapia con broncodilatadores inhalados y los esteroides. Los beta- 2 agonistas de acción corta son de elección, y la asociación con un anticolinérgico en puede ayudar en el control de los síntomas. Los esteroides orales (prednisona 30 - $40 \mathrm{mgr} /$ día) o endovenosos a dosis equivalentes deben usarse por 7 a 10 días, más allá de este tiempo no han mostrado beneficio y aumentan la incidencia de efectos adversos (5, 8). Las infecciones respiratorias están asociadas a un $50-80 \%$ de las EAEPOC (7), hasta el $30 \%$ de estas infecciones pueden ser virales, sin embargo la recomendación actual es el inicio de antibiótico en el paciente que presente aumento en el volumen del esputo asociado a características purulentas del mismo o aumento de la disnea (Anthonisen tipo 1 y 2), esta recomendación se soporta en varios ensayos clínicos que han mostrado que el inicio temprano de terapia antibiótica por 3 a 7 días disminuye la mortalidad a corto plazo y las fallas en el tratamiento $(4,5,6)$.

Los gases arteriales juegan un papel importante en el enfoque y seguimiento del paciente con EAEPOC severa y deben evaluarse de manera especial la saturación arterial de $\mathrm{O}_{2}$, la $\mathrm{PaCO}_{2}$ y el pH. El objetivo debe ser mantener niveles adecuados de $\mathrm{PaO}_{2}$ y $\mathrm{SpO}_{2}$ teniendo en cuenta los valores basales del paciente ya que $\mathrm{PaO}_{2}$ muy por encima de $60 \mathrm{mmHg}$ no confieren beneficio adicional y pueden empeorar la depresión respiratoria y la retención de $\mathrm{CO}_{2}(28)$. El oxígeno suplementario puede administrarse a través de cánula nasal o venturi dependiendo de los requerimientos. Además de la hipoxia se debe presentar atención a la $\mathrm{PaCO}_{2}$ especialmente en presencia de acidosis respiratoria para considerar la necesidad de soporte ventilatorio (Figura 1).

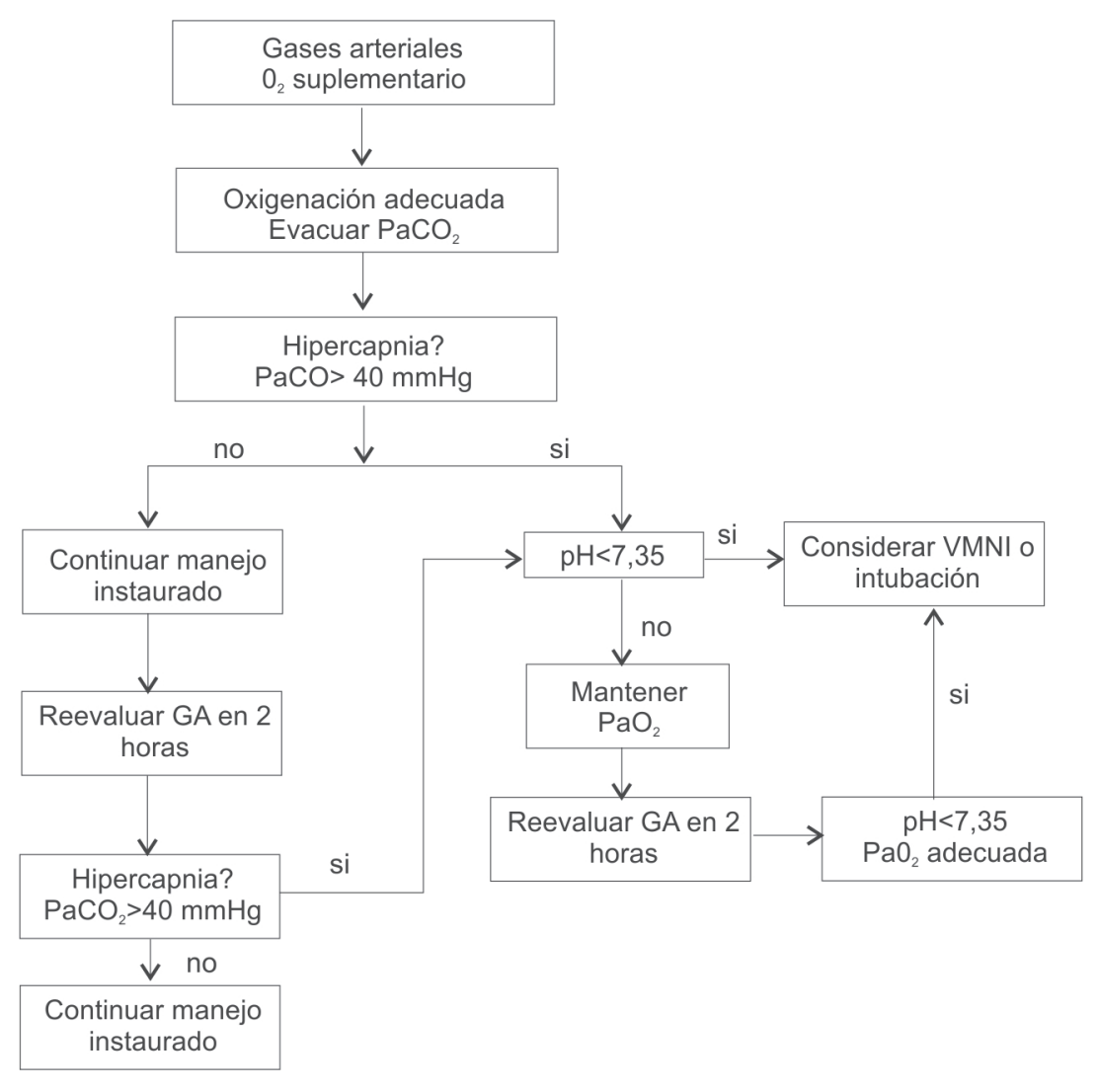

Figura 1. Algoritmo para la evaluación de la acidosis respiratoria en un paciente con EAEPOC. GA: gases ateriales. PaCO : tensión arterial de $\mathrm{CO}_{2}$. $\mathrm{PaO}_{2}$ : tensión arterial de $\mathrm{O}_{2}$. VMNI: ventilación mecánica no invasiva. (Fuente: ATS/ ERS Task Force. Eur Respir J 2004; 23: 932-946) 


\section{ASISTENCIA VENTILATORIA EN EAEPOC}

La asistencia ventilatoria en la EAEPOC severa puede instaurarse a través de dos formas: la VMNI (ventilación mecánica no invasiva) o la intubación orotraqueal (invasiva). Actualmente la VMNI está indicada como la primera opción para pacientes con EAEPOC severa que requieran soporte ventilatorio, siempre y cuando no tengan contraindicaciones para la misma $(1,10)$. La VMNI ha demostrado en ensayos clínicos disminuir la necesidad de intubación orotraqueal, recuperación más rápida de parámetro clínicos y gasimétricos, menor requerimiento de sedación, facilita el retiro del soporte ventilatorio y disminuye de la incidencia de complicaciones como la neumonía asociada al ventilador (11-13).

En este documento vamos a distinguir la VMNI del CPAP (Continuous Positive Airway Pressure) el cual se refiere a la aplicación de una presión positiva continua en la vía aérea a través de todo el ciclo respiratorio usando una máscara facial o nasal en un paciente que está respirando espontáneamente (13). El uso terapéutico del CPAP se basa en mantener un flujo alto de oxígeno en la vía aérea que crea una presión positiva mejorando el reclutamiento alveolar y por lo tanto la oxigenación. A pesar de que el CPAP puede eventualmente mejorar la mecánica respiratoria y la ventilación, debe ser visto como un soporte respiratorio más que ventilatorio ya que los parámetros ventilatorios como la frecuencia respiratoria y el volumen corriente continúan dependiendo exclusivamente del paciente (Figura 2). Además las indicaciones específicas para el uso de CPAP son diferentes a las de la VMNI $(14,15,30)$.
La definición de VMNI implica la provisión de soporte ventilatorio sin instrumentación de la vía aérea (15); el término ventilación fisiológicamente se define como el proceso de intercambio de gases entre el medio ambiente y el alvéolo, a través del cual el oxígeno es llevado de la atmósfera al alvéolo y el dióxido de carbono es liberado desde los alvéolos a la atmósfera. Este proceso está determinado principalmente por tres variables: la frecuencia respiratoria, el volumen corriente y el espacio muerto $(16,17)$.

La fisiopatología de la EAEPOC se caracteriza por un proceso inflamatorio en la vía aérea que causa un disbalance entre la capacidad de trabajo de los músculos respiratorios y la carga respiratoria aumentada (27), dada por broncoconstricción, edema de la vía aérea y aumento en la producción de esputo. Este proceso se comporta como un círculo vicioso en el cual hay un aumento de la resistencia al flujo de aire con compromiso de la ventilación alveolar, hiperinsuflación dinámica, deterioro de la mecánica ventilatoria por restricción de la distensibilidad del sistema y fatiga muscular que Ileva el paciente a falla ventilatoria tipo II o hipercápnica $(15,18)$.

El papel de la ventilación mecánica no invasiva implica una disminución del trabajo respiratorio del paciente a través de una presión positiva expiratoria en la vía aérea (EPAP) usualmente entre $4-8 \mathrm{cmH}_{2} \mathrm{O}$ y una presión soporte ventilatorio o presión positiva inspiratoria (PSV o IPAP) entre $10-20 \mathrm{cmH}_{2} \mathrm{O}$ (figura 3) (19).

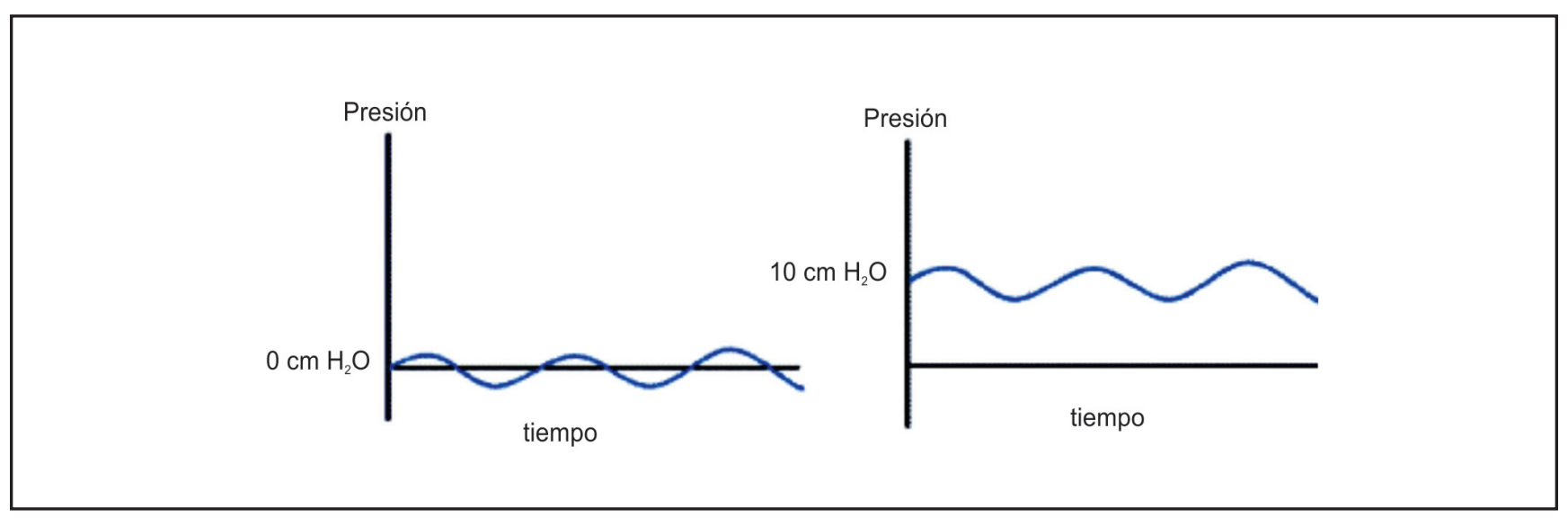

Figura 2. Curvas presión tiempo en respiración espontánea y luego con aplicación de 10 cmH2O de presión positiva continua en la vía aérea. 


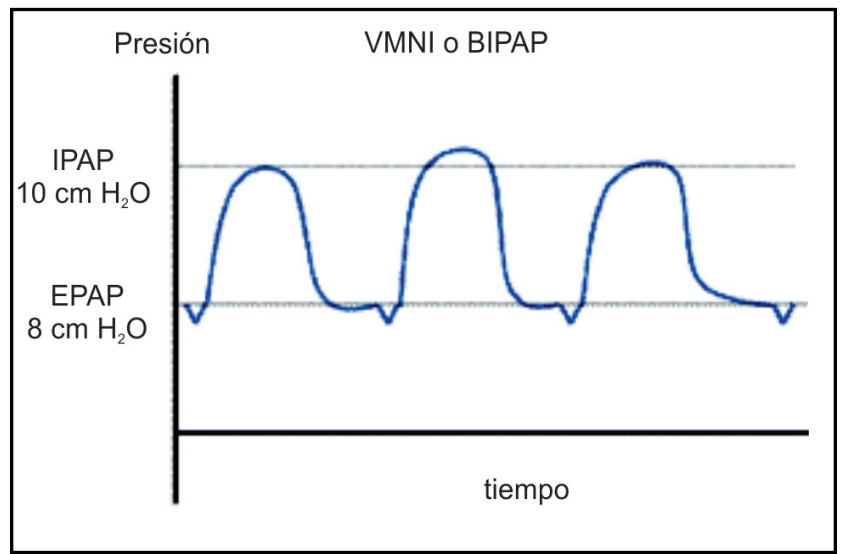

Figura 3. Curva presión tiempo de respiración con VMNI. Compuesto por dos niveles de presión (bilevel o BIPAP), una presión positiva expiratoria o continua (EPAP o CPAP) y una presión de soporte durante la inspiración (IPAP) que es disparada por el paciente a través del descenso de la presión en el sistema cuando éste comienza la inspiración.

Durante la VMNI el esfuerzo inspiratorio del paciente desencadena la entrega de un flujo alto de oxígeno que aumenta la presión en la vía aérea hasta alcanzar un valor predeterminado, esta presión de soporte se mantiene hasta cuando el flujo inspiratorio del paciente cae por debajo de un porcentaje del flujo máximo (usualmente un $30 \%$ ). En consecuencia a pesar de que el paciente recibe un soporte inspiratorio, la frecuencia respiratoria y la duración de la inspiración dependen de la magnitud del esfuerzo inspiratorio. La VMNI también se denomina bilevel o BIPAP ya que el paciente recibe dos niveles de presión soporte, la EPAP y la IPAP, que equivale a un CPAP con presión de soporte inspiratorio $(14,19)$.

El equipo necesario para la VMNI incluye la interface y un ventilador. En cuanto a la interface existen una variedad de máscaras de diferentes diseños y formas, dentro de las más importantes están: la máscara facial completa (full face u oronasal), la facial total, la nasal, las almohadillas nasales y el casco. En cuidado intensivo las más usadas y mejor acopladas al tipo de paciente son la facial completa, facial total y el casco (20).

El uso de VMNI en EAEPOC severa debe considerarse en un paciente que no responde a una terapia médica óptima teniendo en cuenta parámetros clínicos y gasimétricos. Como vimos previamente (figura 1), los gases arteriales son fundamentales en el seguimiento de estos pacientes. Debe considerarse el inicio de VMNI en los siguientes casos $(21,24)$ :

- $\quad$ Hipercapnia $\left(\mathrm{PaCO}_{2}>45 \mathrm{mmHg}\right)$ en presencia de acidosis $(\mathrm{pH}<7,35)$

- $\quad$ Disnea moderada a severa con uso de músculos accesorios
- Frecuencia respiratoria $>25 /$ minuto

Los paciente con acidosis respiratoria severa $(\mathrm{pH}<$ 7,25 ) tienen mayor probabilidad de fracaso terapéutico con VMNI por lo cual debe considerarse en este grupo la intubación orotraqueal como primera opción. Otras contraindicaciones para el uso de VMNI son (20):

- Inestabilidad hemodinámica

- $\quad$ Paro respiratorio

- $\quad$ Secreciones abundantes

- $\quad$ No cooperación del paciente, alteración del estado mental

- Trauma o cirugía cráneo facial o gastroesofágica reciente

- Obesidad extrema

Antes de colocar a un paciente en VMNI el clínico debe tener claro cuál es el plan a seguir y que hacer en caso de fracaso con la VMNI. Una vez iniciada la VMNI debe realizarse un seguimiento que incluya gases arteriales para guiar la toma de decisiones (8):

La evolución clínica y gasimétrica temprana es un predictor del éxito de la VMNI. La ausencia de mejoría en el $\mathrm{pH}$ y la $\mathrm{PaCO}_{2}$ en la primera hora de terapia son factores predictores de fracaso y por lo tanto de la necesidad de intubación así como de mayor mortalidad hospitalaria $(22,24,26)$. La VMNI es una medida temporal de soporte a la patología de base y por lo general se usa de 6 a 24 horas (21) cuando hay adecuada respuesta, de lo contrario, el fracaso puede predecirse desde la primera hora y en este caso hay que definir si el paciente es candidato a intubación. Dentro de las indicaciones para intubación orotraqueal están $(8,25)$ :

- $\quad$ Empeoramiento o no mejoría de $\mathrm{pH}_{\text {y }} \mathrm{PaCO}_{2}$ después de $1-2 \mathrm{~h}$ de $\mathrm{VMNI}$

- Deterioro clínico respiratorio y/o neurológico

- $\quad$ Acidosis e hipercapnia severas $(\mathrm{pH}<7,25 \mathrm{y}$ $\mathrm{PaCO}_{2}>60 \mathrm{mmHg}$ )

- $\quad$ Hipoxemia severa con $\mathrm{PaFI}<20$

- $\quad$ Taquipnea $>35 / \mathrm{min}$

Una vez el paciente está intubado y no hay complicaciones adicionales el objetivo es iniciar el proceso de destete antes de 48 horas y extubar si la respuesta es adecuada, una opción recomendada es el paso a VMNI si el intento de extubación es fallido en el contexto de un paciente con EAEPOC que sufrió falla ventilatoria 


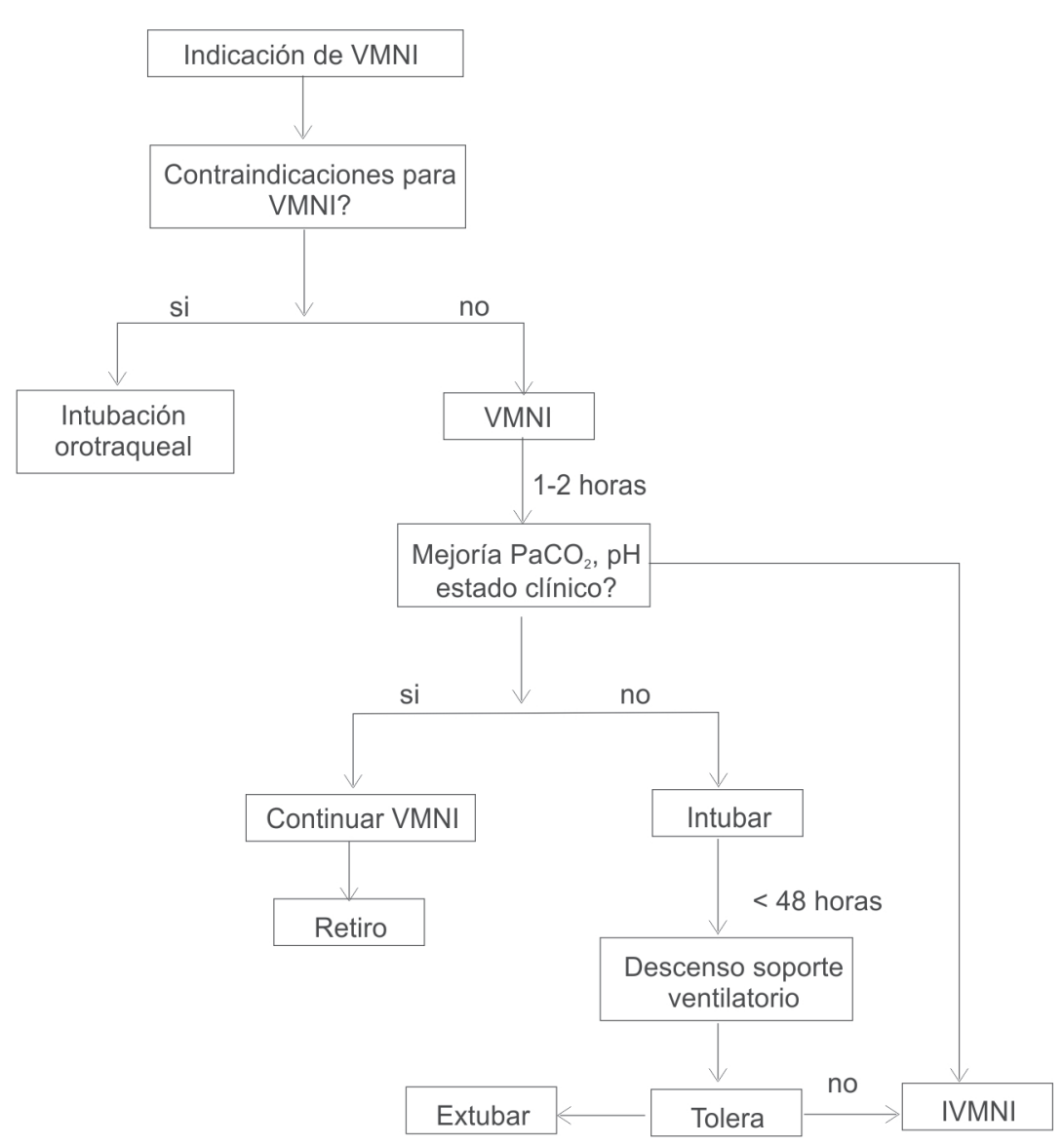

Figura 4. Algoritmo para el uso de VMNI durante EAEPOC. GA: gases ateriales. $\mathrm{PaCO}_{2}$ : tensión arterial de $\mathrm{CO}_{2}$. VMNI: ventilación mecánica no invasiva. (Fuente: ATS/ ERS Task Force. Eur Respir J 2004; 23: 932-946)

hipercápnica, esta estrategia ha demostrado disminuir la necesidad de reintubación, de traqueostomía y mejorar la supervivencia a 90 días $(23,25,26)$.

\section{CONCLUSIÓN}

La EAEPOC severa se relaciona con frecuencia con un manejo irregular de la enfermedad de base y ausencia de rehabilitación cardiopulmonar, además tiene consecuencias sobre el pronóstico y calidad de vida del paciente a corto y largo plazo. Una vez presente, en la EAEPOC severa debe realizarse una adecuada evaluación del paciente que incluya gases arteriales como herramienta fundamental en el enfoque inicial y comparación con estudios posteriores. En los pacientes con requerimiento de asistencia ventilatoria la VMNI es actualmente el método de elección en ausencia de contraindicaciones y su éxito depende de la adecuada selección del paciente, de la interface y un seguimiento clínico y gasimétrico cercano que permita la predicción del éxito o fracaso de la terapia y por lo tanto la toma de conductas adicionales.

\section{REFERENCIAS}

1. Rodríguez-Roisin R. Toward a concensus definition for COPD exacerbation. Chest. 2000;117;398S-401S

2. Garcia-Aymerich J, Barreiro E, Farrero E, Marrades RM, Morera J, Antó JM. Patients hospitalized for COPD have a high prevalence of modifiable risk factors for exacerbation (EFRAM study). Eur Respir J. 2000 Dec;16(6):1037-42.

3. Bahadori K, FitzGerald JM, Levy RD, Fera T, Swinston J. Risk factors and outcomes associates with chronic obstructive pulmonary disease exacerbations requiring hospitalization. Can Res J. 2009; 168: 71-79

4. Rothberg M., Pekow P., et al. Antibiotic Therapy and Treatment Failure in Patients Hospitalized for Acute Exacerbations of Chronic Obstructive Pulmonary Disease. JAMA, May 26, 2010.

5. Daniels JM, Snijders D, de Graaff CS, Vlaspolder F, Jansen HM, Boersma WG. Antibiotics in addition to systemic corticosteroids for acute exacerbations of chronic obstructive pulmonary disease. Am J Respir Crit Care Med. 2010 Jan 15.

6. Anthonisen NR, Manfreda J, et al. 1987. Antibiotic therapy in exacerbations of chronic obstructive pulmonary disease. Ann Intern Med, 106:196-204. 
7. Siddiqi A., Sethi S.. Optimizing antibiotic selection in treating COPD exacerbation. International Journal of COPD 2008:3(1) 31-44.

8. Schuetz P., D. Leuppi, Tamm M., Short versus conventional term glucocorticoid therapy in acute exacerbation of chronic obstructive pulmonary disease. REDUCE trial. Swiss Med Wkly. $2010 ; 140$.

9. ATS/ ERS Task Force. Eur Respir J 2004; 23: 932-946

10. Nava S., Hill N.. Non-invasive ventilation in acute respiratory failure. Lancet . 2009; 374: 250-59.

11. Carrera M., Marín J., Antón A.. A controlled trial of noninvasive ventilation for chronic obstructive pulmonary disease exacerbations. Journal of Critical Care (2009) 24, 473.e7473.e14.

12. G U Meduri, N Abou-Shala, R C Fox, C B Jones, K V Leeper and R G Wunderink. Noninvasive face mask mechanical ventilation in patients with acute hypercapnic respiratory failure. Chest. 1991;100;445-454

13. British Thoracic Society Standards of Care Committee. Noninvasive ventilation in acute respiratory failure. Thorax 2002;57:192-211.

14. Masip J. Ventilación no invasiva en el edema agudo de pulmón. Hipertensión. 2008;25(1):16-22

15. Esmond G., Mikelsons C.. Non invasive respiratory support techniques. 1a edición. Oxford, UK. Blackwell Publishing. 127 152

16. Des Jardins T. Cardiopulmonary anatomy and physiology. $4^{\text {th }}$ edition. Columbia. USA. Delmar/Thomnson learning. 63-115.

17. Levitzky M. Pulmonary Physiology. $7^{\text {th }}$ edition. Mc Graw Hill's ed. USA. Cap. 3.

18. Khilnani G., Banga A. Noninvasive ventilation in patients with chronic obstructive airway disease. International Journal of COPD 2008:3(3) 351-357

19. Mckenzie I. Core Topics in mechanical ventilation. $1^{\text {st }}$ edition. Cambridge. Cambridge University Press. 32 - 53.

20. Elliot M. Non invasive mechanical ventilation. Medicine. 2004. 99 -102 .
21. Antro C, Merico F, Urbino R, Gai V. Non-invasive ventilation as a first-line treatment for acute respiratory failure: "real life» experience in the emergency department. Emerg Med J. 2005 Nov;22(11):772-7

22. Kaya A,Çiledag A, Çayli I, Önen Z, et al. Associated factors with non-invasive mechanical ventilation failure in acute hypercapnic respiratory failure. Tüberküloz ve Toraks Dergisi. 2010; 58(2): 128-134

23. Ferrer M, Sellarés J, Valencia M, Carrillo A. Non-invasive ventilation after extubation in hypercapnic patients with chronic respiratory disorders: randomised controlled trial. Ann Intern Med. 2010 Feb 16; 152(4): JC-24.

24. Khilnani GC, Saikia N, Banga A, Sharma SK. Non-invasive ventilation for acute exacerbation of COPD with very high $\mathrm{PaCO}(2)$ : A randomized controlled trial. Lung India. 2010 Jul;27(3):125-30.

25. Prasad SB, Chaudhry D, Khanna R. Role of noninvasive ventilation in weaning from mechanical ventilation in patients of chronic obstructive pulmonary disease: an Indian experience. Indian J Crit Care Med. 2009 Oct;13(4):207-12

26. Carrera M, Marín JM, Antón A, et al. A controlled trial of noninvasive ventilation for chronic obstructive pulmonary disease exacerbations. J Crit Care. 2009 Sep;24(3):473.e7-14.

27. Purro A, Appendini L, Polillo C, et al. Mechanical determinants of early acute ventilatory failure in COPD patients: a physiologic study. Intensive Care Med. 2009 Apr;35(4):639-47.

28. Joosten SA, Koh MS, Bu X, et al. The effects of oxygen therapy in patients presenting to an emergency department with exacerbation of chronic obstructive pulmonary disease. Med $\mathrm{J}$ Aust. 2007 Mar 5;186(5):235-8.

29. Mohan A, Premanand R, Reddy LN, et al. Clinical presentation and predictors of outcome in patients with severe acute exacerbation of chronic obstructive pulmonary disease requiring admission to intensive care unit. BMC Pulm Med. 2006 Dec 19;6:27.

30. Delclaux C, L'Her E, Alberti C. JAMA. Treatment of acute hypoxemic nonhypercapnic respiratory insufficiency with continuous positive airway pressure delivered by a face mask: A randomized controlled trial. 2000 Nov 8;284(18):2352-60. 